\title{
SOME MORE RECENT RESULTS CONCERNING WEAK ASPLUND SPACES
}

\author{
WARREN B. MOORS
}

Received 13 November 2003

We will present some of the latest advances that have occurred in the study of weak Asplund spaces. In particular, we will give an example of a Gâteaux differentiability space that is not weak Asplund.

\section{Introduction}

In this paper, we will provide some examples of Banach spaces that are Gâteaux differentiability spaces but not weak Asplund, weak Asplund but not in class $(\tilde{\mathscr{Y}})$, in $\operatorname{class}(\tilde{\mathscr{S}})$ but whose dual space is not weak* fragmentable.

We begin with some definitions. A Banach space $X$ is called a weak Asplund space [almost weak Asplund] (Gâteaux differentiability space) if each continuous convex function defined on it is Gâteaux differentiable at the points of a residual [everywhere second category] (dense) subset. While it is easy to see that every weak Asplund space is an almostweak Asplund space and every almost weak Asplund space is a Gâteaux differentiability space, it has been a long standing question as to whether there are in fact Gâteaux differentiability spaces that are not weak Asplund.

In the study of weak Asplund spaces, several classes of topological spaces have played a prominent role; two of which we describe below. A set-valued mapping $\varphi: X \rightarrow 2^{Y}$ acting between topological spaces $X$ and $Y$ is called an usco mapping if for each $x \in X, \varphi(x)$ is a nonempty compact subset of $Y$ and for each open set $W$ in $Y,\{x \in X: \varphi(x) \subseteq W\}$ is open in $X$. An usco mapping $\varphi: X \rightarrow 2^{Y}$ is called a minimal usco if its graph does not contain, as a proper subset, the graph of any other usco defined on $X$.

Below we recall some of the basic properties of minimal uscos.

Proposition 1.1 [2, Proposition 3.1.2]. Let $\varphi: X \rightarrow 2^{Y}$ be an usco acting between topological spaces $X$ and $Y$. Then $\varphi$ is a minimal usco if and only if, for each pair of open subsets $U$ of $X$ and $W$ of $Y$ with $\varphi(U) \cap W \neq \varnothing$, there exists a nonempty open subset $V$ of $U$ such that $\varphi(V) \subseteq W$.

Using this characterisation, one can easily deduce the following facts.

Proposition 1.2 [3, Lemma 2]. Let $\varphi: X \rightarrow 2^{Y}$ be a minimal usco acting between topological spaces $X$ and $Y$. 
(i) If $g: Y \rightarrow Z$ is a continuous mapping into a topological space $Z$, then the mapping $(g \circ \varphi): X \rightarrow 2^{Z}$ defined by $(g \circ \varphi)(x):=\{g(y) \in Z: y \in \varphi(x)\}$ is a minimal usco.

(ii) If $U$ is either a dense subset of $X$ or a nonempty open subset of $X$ then the restriction of $\varphi$, denoted by $\left.\varphi\right|_{U}$, is a minimal usco.

Given a topological space $X$ and a class $\mathscr{C}$ of Baire spaces, we say that $X$ is in Stegall's class with respect to $\mathscr{C}$ if for every $B \in \mathscr{C}$ and minimal usco $\varphi: B \rightarrow 2^{X}, \varphi$ is single valued at some point of $B$. If $\mathscr{C}$ is stable with respect to taking open subspaces and dense Baire subsets (taking open subspaces and dense $G_{\delta}$ subsets), then this is equivalent to that for every $B \in \mathscr{C}$ and minimal usco $\varphi: B \rightarrow 2^{X}, \varphi$ is single-valued at the points of a residual (everywhere second category) subset of $B,[3$, Proposition 1$]$.

When $\mathscr{C}$ is the class of all Baire (all complete metric) spaces, we simply say that $X$ is a Stegall (weakly Stegall) space. For us, the significance of this class of topological spaces stems from the fact that for a Banach space $X$ if $\left(X^{*}\right.$, weak $\left.{ }^{*}\right)$ is a Stegall (weakly Stegall) space, then $X$ is weak Asplund, [2, Theorem 3.2.2] (almost weak Asplund, [6, Theorem 13]).

Note. We will say that a Banach space $X$ belongs to $\operatorname{class}(\tilde{\mathscr{Y}})[\operatorname{class}(w \tilde{\mathscr{S}})]$ if $\left(X^{*}\right.$, weak $\left.^{*}\right)$ is a Stegall space [weakly Stegall space].

The other class of topological spaces that we will consider in this paper is the class of fragmentable spaces. A topological space $X$ is said to be fragmentable if there exists a metric $d$ on $X$ such that for each $\varepsilon>0$ and nonempty subset $Y$ of $X$, there exists an open set $U$ in $X$ such that (i) $Y \cap U \neq \varnothing$ and (ii) $d-\operatorname{diam}(Y \cap U)<\varepsilon$.

As with Stegall spaces, our interest in these spaces emanates from the fact that for a Banach space $X$ if $\left(X^{*}\right.$, weak $\left.{ }^{*}\right)$ is fragmentable, then $X$ is weak Asplund (in fact, if $\left(X^{*}\right.$, weak $\left.^{*}\right)$ is fragmentable, then $\left(X^{*}\right.$, weak $\left.{ }^{*}\right)$ is in Stegall's class, [2, Theorem 5.2.2]).

Although the exact relationship between fragmentable spaces, Stegall spaces, weak Asplund spaces, and Gâteaux differentiability spaces remains unclear, several partial results are known. For instance, the authors in [5] have provided an example (with the aid of some additional set-theoretic assumptions) of a Banach space $X$ such that $\left(X^{*}\right.$, weak $\left.{ }^{*}\right)$ is in Stegall's class but is not fragmentable while in [4] the author has given an example (also with the aid of some additional set-theoretic assumptions) of a Banach space $X$ such that $X$ is weak Asplund but $\left(X^{*}\right.$, weak $\left.^{*}\right)$ is not in Stegall's class. Moreover, in this paper we give an example (in ZFC) of a Gâteaux differentiability space that is not weak Asplund.

One of the interesting aspects of all these examples is that they are all based upon the following class of topological spaces.

\section{Kalenda compacta}

Let $A$ be an arbitrary subset of $(0,1)$ and let

$$
K_{A}:=[(0,1] \times\{0\}] \cup[(\{0\} \cup A) \times\{1\}]
$$

If we equip this set with the order topology generated by the lexicographical (dictionary) ordering (i.e., $\left(s_{1}, s_{2}\right) \leq\left(t_{1}, t_{2}\right)$ if and only if either $s_{1}<t_{1}$ or $s_{1}=t_{1}$ and $\left.s_{2} \leq t_{2}\right)$, then with this topology $K_{A}$ is a compact Hausdorff space [3, Proposition 2], which we will call 
the Kalenda compact associated with the set $A$. In the special case of $A=(0,1)$, the Kalenda compact $K_{A}$ reduces to the well-known "double arrow" space.

Many of the basic properties of the Kalenda compacta may be found in [3]. In particular, the following results may be found there.

Theorem 2.1 [3, Proposition 3]. Let $A$ be an arbitrary subset of $(0,1)$. Then the following properties are equivalent:

(i) A is countable;

(ii) $K_{A}$ is metrizable;

(iii) $K_{A}$ is fragmentable.

Theorem 2.2 [3, Proposition 5]. Let $A$ be an arbitrary subset of $(0,1)$. Then the following properties are equivalent:

(i) every closed subspace of $K_{A}$ contains a dense completely metrizable subspace;

(ii) $A$ is perfectly meagre.

We recall that a subset $A \subseteq \mathbb{R}$ is called perfectly meagre if for every perfect subset $P \subseteq \mathbb{R}$ the intersection $A \cap P$ is meagre (i.e., first category) in $P$.

For a compact Hausdorff space $K$, we will denote by $\mathcal{M}(K)\left\{\mathcal{M}^{+}(K)\right\}\left[\mathcal{M}_{1}^{+}(K)\right]$ the space of all Radon measures on $K$ \{positive Radon measure on $K\}$ [positive Radon measures on $K$, with total mass at most one], equipped with the weak topology induced by the continuous real-valued functions defined on $K$. By Riesz's representation theorem, we know that $M(K)\left\{\mathcal{M}^{+}(K)\right\}\left[\mathcal{M}_{1}^{+}(K)\right]$ is homeomorphic to $C(K)^{*}$ the positive linear functionals in $\left.C(K)^{*}\right\}$ [the positive linear functionals in $C(K)^{*}$ with norm at most one], equipped with the weak* topology.

2.1. Single-valuedness of minimal uscos into $M_{1}^{+}\left(K_{A}\right)$. The goal of this section is to present some sufficient conditions for a minimal usco acting from a Baire space into $M_{1}^{+}\left(K_{A}\right)$ to be single valued. To accomplish this we will need several (four in fact) technical results.

For an arbitrary subset $A$ of $(0,1)$, we will denote by $\pi_{A}$ the natural projection of $K_{A}$ onto $[0,1]$, defined by $\pi_{A}(t, \varepsilon):=t$, and we will denote by $\pi_{A}^{* *}$ the natural projection of $M_{1}^{+}\left(K_{A}\right)$ onto $M_{1}^{+}([0,1])$, defined by $\pi_{A}^{* *}(\mu)(E):=\mu\left(\pi_{A}^{-1}(E)\right)$ for each Borel subset $E$ of $[0,1]$. In both cases the mappings are continuous.

Lemma 2.3. Let $A$ be an arbitrary subset of $(0,1)$ and suppose that $\mu, v \in M_{1}^{+}\left(K_{A}\right)$. Then $\mu=\nu$ if (and only if) $\pi_{A}^{* *}(\mu)=\pi_{A}^{* *}(\nu)$ and the restrictions of $\mu$ and $\nu$ to $\pi_{A}^{-1}(t)$ coincide for each $t \in A$.

Proof. Since the functions in $C\left(K_{A}\right)$ separate the measures in $M_{1}^{+}\left(K_{A}\right)$, it is sufficient to show that

$$
\int_{K_{A}} f \mathrm{~d} \mu=\int_{K_{A}} f \mathrm{~d} \nu \quad \text { for each } f \in C\left(K_{A}\right) .
$$

To this end, fix $f \in C\left(K_{A}\right)$ and define $g:[0,1] \rightarrow \mathbb{R}$ by

$$
g(t):= \begin{cases}f(t, 1) & \text { if } t=0, \\ f(t, 0) & \text { if } t \in(0,1]\end{cases}
$$


Then $g$ is Borel measurable and $J:=\{t \in A: g(t) \neq f(t, 1)\}$ is countable. In particular, this means that $\mu$ and $\nu$ coincide on $\pi_{A}^{-1}(J)$. On the other hand, $f=g \circ \pi_{A}$ on $K_{A} \backslash \pi_{A}^{-1}(J)$ and so

$$
\begin{aligned}
\int_{K_{A}} f \mathrm{~d} \mu & =\int_{K_{A} \backslash \pi_{A}^{-1}(J)}\left(g \circ \pi_{A}\right) \mathrm{d} \mu+\int_{\pi_{A}^{-1}(J)} f \mathrm{~d} \mu \\
& =\int_{[0,1] \backslash J} g \mathrm{~d}\left(\pi_{A}^{* *}(\mu)\right)+\int_{\pi_{A}^{-1}(J)} f \mathrm{~d} \mu \\
& =\int_{[0,1] \backslash J} g \mathrm{~d}\left(\pi_{A}^{* *}(\nu)\right)+\int_{\pi_{A}^{-1}(J)} f \mathrm{~d} \nu \\
& =\int_{K_{A} \backslash \pi_{A}^{-1}(J)}\left(g \circ \pi_{A}\right) \mathrm{d} \nu+\int_{\pi_{A}^{-1}(J)} f \mathrm{~d} \nu \\
& =\int_{K_{A}} f \mathrm{~d} \nu .
\end{aligned}
$$

Lemma 2.4. Let $A$ be an arbitrary subset of $(0,1)$ and let $\varphi: B \rightarrow 2^{M_{1}^{+}\left(K_{A}\right)}$ be a minimal usco defined on a Baire space $B$. Then there exists a dense $G_{\delta}$ subset $G$ of $B$ and continuous functions $\left(g_{n}: n \in \mathbb{N}\right)$ from $G$ into $[0,1]$ such that for each $x \in G, \pi_{A}^{* *}(\varphi(x))$ is a singleton and

$$
\left\{t \in[0,1]: \pi_{A}^{* *}(\mu)(\{t\})=\mu\left(\pi_{A}^{-1}(t)\right)>0 \text { for some } \mu \in \varphi(x)\right\} \subseteq\left\{g_{n}(x): n \in \mathbb{N}\right\} .
$$

Proof. Fix for a moment $\varepsilon>0$, a closed set $F$, and an open set $U$ with $\varnothing \neq F \subseteq U \subseteq[0,1]$. Let $Q_{(\varepsilon, F, U)}$ denote the set of all those $\mu \in \mathcal{M}_{1}^{+}\left(K_{A}\right)$ for which there is a $\xi \in F$ such that

$$
\pi_{A}^{* *}(\mu)(U \backslash\{\xi\})=\mu\left(\pi_{A}^{-1}(U \backslash\{\xi\})\right) \leq \varepsilon<2 \varepsilon \leq \mu\left(\pi_{A}^{-1}(\xi)\right)=\pi_{A}^{* *}(\mu)(\{\xi\}) .
$$

Then $Q_{(\varepsilon, F, U)}$ is closed and for each $\mu \in Q_{(\varepsilon, F, U)}$ there is exactly one $\xi:=\xi(\mu) \in F$ with the above property. Moreover, the mapping $\mu \mapsto \xi(\mu)$ from $Q_{(\varepsilon, F, U)}$ into $[0,1]$ is continuous. Hence, by Tietze's extension theorem, this mapping has an extension to $\mathcal{M}_{1}^{+}\left(K_{A}\right)$, which we call $\xi_{(\varepsilon, F, U)}$. By Proposition 1.2(i) and the fact that $[0,1]$ lies in Stegall's class, we have that $\xi_{(\varepsilon, F, U)} \circ \varphi$ is single-valued (and continuous) at the points of a dense $G_{\delta}$ subset $G_{(\varepsilon, F, U)}$ of $B$. Let $\mathscr{B}$ be a countable base for the topology on $[0,1]$ and let

$$
G:=\bigcap\left\{G_{(\varepsilon, \bar{V}, U)}: \varepsilon \in(0, \infty) \cap \mathbb{Q}, V, U \in \mathscr{B} \text { and } \varnothing \neq V \subseteq \bar{V} \subseteq U\right\}
$$

Then if we denote by $\left(g_{n}: n \in \mathbb{N}\right)$ the functions (though technically they are single-valued set-valued mappings) in

$$
\left\{\left.\left(\xi_{(\varepsilon, \bar{V}, U)} \circ \varphi\right)\right|_{G}: \varepsilon \in(0, \infty) \cap \mathbb{Q}, V, U \in \mathscr{B} \text { and } \varnothing \neq V \subseteq \bar{V} \subseteq U\right\}
$$

ordered into a sequence, then we have the following. If $x \in G, t \in[0,1]$ and $\mu \in \varphi(x)$ are such that $\mu\left(\pi_{A}^{-1}(t)\right)>0$, then $\{t\}=\left.\left(\xi_{(\varepsilon, \bar{V}, U)} \circ \varphi\right)\right|_{G}(x)$ for some $(\varepsilon, \bar{V}, U)$ and so $t=g_{n}(x)$ for some $n \in \mathbb{N}$. Moreover, by Proposition 1.2(i) and the fact that $\mathcal{M}_{1}^{+}([0,1])$ is metrizable (and in particular in Stegall's class), we may assume, after possibly making $G$ smaller, that $\pi_{A}^{* *} \circ \varphi$ is single valued on $G$. 
The following theorem is essentially a consequence of Lemmas 2.3 and 2.4.

Theorem 2.5. Let $A$ be an arbitrary subset of $(0,1)$ and let $\varphi: B \rightarrow 2^{M_{1}^{+}\left(K_{A}\right)}$ be a minimal usco defined on a Baire space B. If $\left(g_{n}: n \in \mathbb{N}\right)$ and $G$ are the continuous functions and $G_{\delta}$ subset of B given in Lemma 2.4, then $\varphi$ is single valued at $x \in G$ if for each $n \in \mathbb{N}$, the restriction of all the measures in $\varphi(x)$ to $\pi_{A}^{-1}\left(g_{n}(x)\right)$ coincide.

To achieve our goal in Section 3, we need two more results.

We will say that a subset $Y$ of a topological space $X$ has countable separation in $X$ if there is a countable family $\left\{C_{n}: n \in \mathbb{N}\right\}$ of closed subsets of $X$ such that for every pair $\{x, y\}$ with $y \in Y$ and $x \in X \backslash Y,\{x, y\} \cap C_{n}$ is a singleton for at least one $n \in \mathbb{N}$. If we denote by $X_{\Sigma}$ the family of all subsets of $X$ with countable separation in $X$, then $X_{\Sigma}$ is a $\sigma$-algebra that contains all the open subsets of $X$. Moreover, $X_{\Sigma}$ is closed under the Souslin operation. For a mapping $g: X \rightarrow Y$ acting between topological spaces $X$ and $Y$, we will say that $g$ is separation measurable if $g^{-1}(U)$ has countable separation in $X$ for each open set $U$ in $Y$. If the range space $Y$ has countable separation weight (i.e., there exists a countable open cover $\mathcal{U}$ of $Y$ such that for each $y \in Y, \bigcap\{O \in \mathcal{U}: y \in O\}=\{y\}$ ), then separation-measurable mappings have single-valuedness implications for minimal usco mappings.

Lemma 2.6. Let $\varphi: B \rightarrow 2^{X}$ be a minimal usco acting from a Baire space $B$ into a topological space $X$ and let $g: X \rightarrow Y$ be a separation measurable mapping acting from $X$ into a topolog$i$ cal space $Y$ with countable separation weight. Then $(g \circ \varphi): B \rightarrow 2^{Y}$ is single valued at the points of a residual subset of $B$. In particular, if $g$ is Borel measurable and $Y$ is a separable metric space, then $(g \circ \varphi): B \rightarrow 2^{Y}$ is single valued at the points of a residual subset of $B$.

Proof. Let $U:=\left\{U_{m}: m \in \mathbb{N}\right\}$ be an open cover of $Y$ that separates the points of $Y$. For each $m \in \mathbb{N}$, let $\left\{C_{(m, n)}: n \in \mathbb{N}\right\}$ be a countable family of closed subsets of $X$ that "separate" $g^{-1}\left(U_{m}\right)$ from $X \backslash g^{-1}\left(U_{m}\right)$ (i.e., if $x \in g^{-1}\left(U_{m}\right)$ and $y \notin g^{-1}\left(U_{m}\right)$, then there exists an $n \in \mathbb{N}$ such that $\left.\left|C_{(m, n)} \cap\{x, y\}\right|=1\right)$. For each $(m, n) \in \mathbb{N}^{2}$, consider the dense open set

$$
O_{(m, n)}:=B \backslash \varphi^{-1}\left(C_{(m, n)}\right) \cup \operatorname{int} \varphi^{-1}\left(C_{(m, n)}\right)=B \backslash \partial\left[\varphi^{-1}\left(C_{(m, n)}\right)\right]
$$

By the minimality of $\varphi$ (see Proposition 1.1),

$$
O_{(m, n)} \subseteq\left\{x \in B: \varphi(x) \cap C_{(m, n)}=\varnothing \text { or } \varphi(x) \subseteq C_{(m, n)}\right\}
$$

Note that if $x \in \bigcap_{n \in \mathbb{N}} O_{(m, n)}$ then either $\varphi(x) \subseteq g^{-1}\left(U_{m}\right)$ or $\varphi(x) \cap g^{-1}\left(U_{m}\right)=\varnothing$. Let $R:=$ $\bigcap\left\{O_{(m, n)}:(m, n) \in \mathbb{N}^{2}\right\}$. We will complete the proof by showing that $g \circ \varphi$ is single valued at the points of $R$. To see this, consider $x \in R$ and suppose that $g \circ \varphi$ is not single-valued at $x$. Then there exists an $m \in \mathbb{N}$ such that

$$
\varnothing \neq \varphi(x) \cap g^{-1}\left(U_{m}\right) \neq \varphi(x) .
$$

But this is impossible since $x \in \bigcap_{n \in \mathbb{N}} O_{(m, n)}$. Hence $g \circ \varphi$ is single valued on $R$. 


\section{Some more recent results concerning weak Asplund spaces}

LEMMA 2.7. Let $A$ be an arbitrary subset of $(0,1), \varphi: B \rightarrow 2^{\mathcal{M}_{1}^{+}\left(K_{A}\right)}$ a minimal usco defined on a Baire space $B$, and $g: B \rightarrow[0,1]$ a locally constant mapping. Then there exists a dense $G_{\delta}$ subset $G$ of $B$ such that for each $x \in G$ the restriction of all the measures in $\varphi(x)$ to $\pi_{A}^{-1}(g(x))$ coincide.

Proof. Let $\mathcal{U}:=\left\{U_{\alpha}: \alpha \in I\right\}$ be a maximal collection of nonempty disjoint open subsets of $B$ such that $g$ is constant of each $U_{\alpha}, \alpha \in I$. Such a maximal family exists by Zorn's lemma and it is easy to check that $U:=\bigcup_{\alpha \in I} U_{\alpha}$ is dense in $B$. Now, for each $\alpha \in I$, choose $x_{\alpha} \in U_{\alpha}$. Then since the restriction mapping $\rho_{\pi_{A}^{-1}\left(g\left(x_{\alpha}\right)\right)}$ from $M_{1}^{+}\left(K_{A}\right)$ into $\mathcal{M}_{1}^{+}\left(\pi_{A}^{-1}\left(g\left(x_{\alpha}\right)\right)\right)$ is Borel measurable, we have from Lemma 2.6 that $\rho_{\pi_{A}^{-1}\left(g\left(x_{\alpha}\right)\right)} \circ \varphi$ is singlevalued at the points of a dense $G_{\delta}$ subset $G_{\alpha}$ of $U_{\alpha}$. Thus, $G:=\bigcup G_{\alpha}$ is the required dense $G_{\delta}$ set of $B$.

\section{Distinguishing the Kalenda compacta}

In this section of the paper, we will characterise, in terms of the set $A$, when $\left(C\left(K_{A}\right)^{*}\right.$, weak $\left.^{*}\right)$ lies in Stegall's class. We will also provide an example of a set $A$ for which $C\left(K_{A}\right)$ is a non-weak Asplund Gâteaux differentiability space.

In the proof of the next theorem, we will need the following basic properties of Stegall spaces. Since the proofs of these assertions are identical to those given in [2, Theorem 3.1.5], we will not repeat them here.

Proposition 3.1. Let $X$ and $Y$ be topological spaces and let $\mathscr{C}$ be a class of Baire spaces that is stable with respect to taking open subspaces and dense Baire subsets.

(i) Let $g: X \rightarrow Y$ be perfect mapping onto $Y$. If $X$ is a Stegall space with respect to $\mathscr{b}$ then $Y$ is a Stegall space with respect to $\mathscr{C}$.

(ii) Let $\left\{X_{n}: n \in \mathbb{N}\right\}$ be cover of $X$. If each $X_{n}$ is closed and in Stegall's class with respect to $\mathscr{C}$, then $X$ is a Stegall space with respect to $\mathscr{C}$.

(iii) If $\left\{X_{n}: n \in \mathbb{N}\right\}$ are Stegall spaces with respect to $\mathscr{C}$, then $\prod_{n=1}^{\infty} X_{n}$ is a Stegall space with respect to $\mathscr{C}$.

By combining Riesz's representation theorem with Proposition 3.1, we obtain the following fact.

Corollary 3.2. Let $\mathscr{C}$ be a class of Baire spaces that is stable with respect to taking open subspaces and dense Baire subsets. Then for a compact Hausdorff space $K,\left(C(K)^{*}\right.$, weak $\left.{ }^{*}\right)$ is a Stegall space with respect to $\mathscr{C}$ if and only if $M_{1}^{+}(K)$ is a Stegall space with respect to $\mathscr{C}$.

Theorem 3.3 [4, proposition]. Let $\mathscr{C}$ be a class of Baire metric spaces that is stable with respect to taking open subspaces and dense Baire subsets and let $A$ be an arbitrary subset of $(0,1)$. Then the following assertions are equivalent:

(i) $\left(C\left(K_{A}\right)^{*}\right.$, weak $\left.{ }^{*}\right)$ is in Stegall's class with respect to $\mathscr{C}$;

(ii) $K_{A}$ is in Stegall's class with respect to $\mathscr{C}$;

(iii) for any $B \in \mathscr{C}$ and any continuous function $g: B \rightarrow A$, the function $g$ has at least one local minimum or local maximum;

(iv) for any $B \in \mathscr{C}$ and any continuous function $g: B \rightarrow A$, there is a nonempty open set $U \subseteq B$ such that $g$ is constant on $U$. 
Proof. (i) $\Rightarrow$ (ii). This follows from the fact that $K_{A}$ is homeomorphic to a closed subspace of $\left(C\left(K_{A}\right)^{*}\right.$, weak $\left.{ }^{*}\right)$.

(ii) $\Rightarrow$ (iii). Suppose (ii) holds. Let $B \in \mathscr{C}$ and let $g: B \rightarrow A$ be a continuous function. In order to obtain a contradiction, we assume that $g$ has no local extrema. Then the mapping $\varphi: B \rightarrow 2^{K_{A}}$ defined by, $\varphi(t):=\{g(t)\} \times\{0,1\}$ is not only an usco but in fact a minimal usco. Therefore, since $K_{A}$ is in the class of Stegall spaces with respect to $\mathscr{b}$, we have our desired contradiction since $\varphi$ is everywhere two valued.

(iii) $\Rightarrow$ (iv). Suppose that (iii) holds and that there is some $B \in \mathscr{C}$ and some continuous function $g: B \rightarrow A$ that is not constant on any nonempty open subset of $B$. Fix a metric $\rho$ generating the topology of $B$. For each $n \in \mathbb{N}$, define

$$
\begin{aligned}
& E_{n}^{\max }:=\left\{x \in B: g(x)=\max \left\{g\left(x^{\prime}\right): \rho\left(x, x^{\prime}\right)<\frac{1}{n}\right\}\right\} ; \\
& E_{n}^{\min }:=\left\{x \in B: g(x)=\min \left\{g\left(x^{\prime}\right): \rho\left(x, x^{\prime}\right)<\frac{1}{n}\right\}\right\} .
\end{aligned}
$$

Then clearly both of the sets $E_{n}^{\max }$ and $E_{n}^{\min }$ are closed and $E:=\bigcup_{n \in \mathbb{N}}\left[E_{n}^{\max } \cup E_{n}^{\min }\right]$ is the set of all local extrema of $g$ on $B$. If one of the sets $E_{n}^{\max }$ or $E_{n}^{\min }$ has an interior point, then $g$ is constant on a neighbourhood of it. Indeed, if $x$ is an interior point of $E_{n}^{\max }$, then $B_{\rho}(x ; \delta) \subseteq E_{n}^{\max }$ for some $0<\delta<1 / n$. Let $x^{\prime} \in B(x ; \delta)$. Then both $g(x) \geq g\left(x^{\prime}\right)$ and $g\left(x^{\prime}\right) \geq g(x)$ hold and so $g(x)=g\left(x^{\prime}\right)$, which shows that $g$ is constant on $B(x ; \delta)$. Hence both of the sets $E_{n}^{\max }$ and $E_{n}^{\min }$ are closed and nowhere dense. Therefore $E$ is a firstcategory set and $B^{\prime}:=B \backslash E$ is a dense Baire subspace of $B$ and so it belongs to $\mathscr{C}$. Thus, by (iii), $\left.g\right|_{B^{\prime}}$ has a local extremum at a point $x \in B^{\prime}$. Then, by continuity of $g$ and density of $B^{\prime}$ in $B, g$ has a local extremum at $x$, with respect to $B$, too. Thus $x \in E$ and hence we have a contradiction.

(iv) $\Rightarrow$ (i). By Corollary 3.2, it is sufficient to show that $\mathcal{M}_{1}^{+}\left(K_{A}\right)$ is in Stegall's class with respect to $\mathscr{C}$. To this end, let $B \in \mathscr{C}$ and let $\varphi: B \rightarrow 2^{\mathcal{M}_{1}^{+}\left(K_{A}\right)}$ be a minimal usco. Furthermore, let $\left(g_{n}: n \in \mathbb{N}\right)$ and $G$ be the continuous functions and dense $G_{\delta}$ subset of $B$ given in Lemma 2.4. Since $G \in \mathscr{C}$ and the restriction of $\varphi$ to $G$ remains a minimal usco (see, Proposition 1.2(ii)), we see that there is no loss of generality in assuming that $B=G$. Now, by Theorem 2.5, it is sufficient to show that for each $n \in \mathbb{N}$, the set

$$
G_{n}:=\left\{x \in B: \text { the restriction of all the measures in } \varphi(x) \text { to } \pi_{A}^{-1}\left(g_{n}(x)\right) \text { coincide }\right\}
$$

is residual in $B$. To accomplish this, we fix $n \in \mathbb{N}$ and let $\mathscr{G}_{n}$ be the union of all the open subsets of $B$ on which $g_{n}$ is constant and let $\mathscr{H}_{n}:=B \backslash \overline{\mathscr{G}_{n}}$. By Lemma 2.7 we know that $\mathscr{G}_{n} \backslash G_{n}$ is first category in $B$. Hence we need only to show that $\mathscr{H}_{n} \backslash G_{n}$ is first category in $B$. In fact, since $\mathscr{H}_{n} \backslash G_{n} \subseteq g_{n}^{-1}(A) \cap \mathscr{H}_{n}$, we need only to show that $g_{n}^{-1}(A) \cap \mathscr{H}_{n}$ is first category in $B$. Thus, in order to obtain a contradiction, we assume that $g_{n}^{-1}(A) \cap \mathscr{H}_{n}$ is second category in $B$. Then, by [2, Proposition 3.2.5], there exists a nonempty open subset $U$ in $\mathscr{H}_{n}$ such that $U \cap g_{n}^{-1}(A)$ is a dense Baire subset of $U$. Therefore, $U \cap g_{n}^{-1}(A)$ belongs to $\mathscr{C}$ and so by (iv) there is a nonempty open $V$ of $U$ such that $\left.g_{n}\right|_{\left(U \cap g_{n}^{-1}(A)\right)}$ is constant on $V \cap\left(U \cap g_{n}^{-1}(A)\right)=V \cap g_{n}^{-1}(A)$. Then, by the continuity of $g_{n}$ and the density of 
$g_{n}^{-1}(A) \cap V$ in $V, g_{n}$ is constant on $V$. However, $V \cap \mathscr{G}_{n}=\varnothing$ and so we have our desired contradiction.

Let $A$ be an arbitrary subset of $(0,1)$ and let $\mathscr{C}$ be a class of Baire metric spaces. Then we will say that a subset $A$ of $(0,1)$ satisfies property $(*)$ with respect to $\mathscr{C}$ if for every $B \in \mathscr{C}$ and every continuous function $f: B \rightarrow A$ there exists a nonempty open set $U$ of $B$ such that $f$ is constant on $U$.

Corollary 3.4 [4, Theorem 1]. (i) If there is an uncountable subset $A$ of $(0,1)$ that satisfies property $(*)$ with respect to the class of all Baire metric spaces, then $\left(C\left(K_{A}\right)^{*}\right.$, weak $\left.{ }^{*}\right)$ belongs to Stegall's class but is not fragmentable.

(ii) If there is an uncountable subset $A$ of $(0,1)$ that satisfies property $(*)$ with respect to the class of all Baire metric spaces of density at most card $(A)$, then $C\left(K_{A}\right)$ is a weak Asplund space but $\left(C\left(K_{A}\right)^{*}\right.$, weak $\left.{ }^{*}\right)$ is not fragmentable.

(iii) If there is a subset $A$ of $(0,1)$ that satisfies property $(*)$ with respect to the class of all Baire metric spaces of density at most $\operatorname{card}(A)$, but not property $(*)$ with respect to the class of all Baire metric spaces, then $C\left(K_{A}\right)$ is a weak Asplund space but $\left(C\left(K_{A}\right)^{*}\right.$, weak $\left.{ }^{*}\right)$ is not in Stegall's class.

Proof. (i) From Theorem 2.1, it follows that $K_{A}$ is not fragmentable. On the other hand, it is shown in [5, Theorem 3] that for a Banach space $X,\left(X^{*}\right.$, weak $\left.^{*}\right)$ is in Stegall's class if and only if it is in Stegall's class with respect to the class of all Baire metric spaces. The result then follows from Theorem 3.3.

(ii) Again from Theorem 2.1 it follows that $K_{A}$ is not fragmentable. To show that $C\left(K_{A}\right)$ is weak Asplund, we need the result [2, Theorem 3.2.2] that for a Banach space $X$ if $\left(X^{*}\right.$, weak $\left.^{*}\right)$ is in the class of Stegall spaces with respect to the class of all Baire metric spaces with density at most equal to the density of $X$, then $X$ is weak Asplund. The result then follows from Theorem 3.3 and the fact that the density of $C\left(K_{A}\right)$ equals $\operatorname{card}(A)$.

(iii) As mentioned in part (ii), if $\left(C\left(K_{A}\right)^{*}\right.$, weak $\left.{ }^{*}\right)$ is in the class of Stegall spaces with respect to the class of all Baire metric spaces with density at most equal to the density of $C\left(K_{A}\right)$, then $C\left(K_{A}\right)$ is weak Asplund. The fact that $\left(C\left(K_{A}\right)^{*}\right.$, weak $\left.{ }^{*}\right)$ is not a Stegall space follows directly from Theorem 3.3.

Remark 3.5. Up to this point, we have not dwelt upon the question of whether there are in fact subsets of $(0,1)$ that satisfy any of the hypotheses of Corollary 3.4. For a discussion on this see $[3,4]$. We mention here, though, that in all cases additional set-theoretic axioms are required.

If $\mathscr{A}$ is a proper $\sigma$-ideal of subsets of $2^{\mathbb{N}}$ and $N$ is a subset of a metric space $M$, then we will say that $N$ is $\mathscr{A}$-negligible if $\gamma^{-1}(N) \in \mathscr{A}$ for each $\gamma$ belonging to a residual subset $R_{N}$ of $C\left(2^{\mathbb{N}} ; M\right)$-the continuous functions from $2^{\mathbb{N}}$ into $M$ equipped with the topology of uniform convergence.

Note. The residual set $R_{N}$ will in general depend upon the set $N$.

For each $n \in \mathbb{N}$ and $t \in 2^{n}$, we define $C_{t}:=\left\{t^{\prime} \in 2^{\mathbb{N}}:\left.t^{\prime}\right|_{n}=t\right\}$ and $C_{\varnothing}:=2^{\mathbb{N}}$. Further, for each $n \in \mathbb{N}$, we will let $\Gamma_{n}:=\left\{\gamma \in C\left(2^{\mathbb{N}} ; M\right): \gamma\right.$ is constant on $C_{t}$ for each $\left.t \in 2^{n}\right\}$. A simple compactness argument shows that for each $n \in \mathbb{N}, \bigcup_{k \geq n} \Gamma_{k}$ is dense in $C\left(2^{\mathbb{N}} ; M\right)$. 
Lemma 3.6. Let $(M, d)$ be a metric space. (i) If $U$ is a dense open subset of $M$, then $\{\gamma \in$ $\left.C\left(2^{\mathbb{N}} ; M\right): \gamma\left(2^{\mathbb{N}}\right) \subseteq U\right\}$ is a dense open subset of $C\left(2^{\mathbb{N}} ; M\right)$. (ii) If $g: U \rightarrow X$ is a continuous function acting from a nonempty open subset $U$ of $M$ into a completely regular topological space $X$ that is not constant on any nonempty open subset of $U$, then there exists a residual subset of $C\left(2^{\mathbb{N}} ; M\right)$ such that for each $\gamma$ in this set, $g \circ \gamma$ is 1 -to-1 on $\gamma^{-1}(U)$ and $\gamma^{-1}(U)$ is clopen (i.e., both open and closed) in $2^{\mathbb{N}}$.

Proof. (i) Firstly, it is easy to see that $\left\{\gamma \in C\left(2^{\mathbb{N}} ; M\right): \gamma\left(2^{\mathbb{N}}\right) \subseteq U\right\}$ is open in $C\left(2^{\mathbb{N}} ; M\right)$. Thus, it remains to show that it is dense. For each $n \in \mathbb{N}$, let $\Gamma_{n}(U):=\left\{\gamma \in \Gamma_{n}: \gamma\left(2^{\mathbb{N}}\right) \subseteq\right.$ $U\}$. It follows from the density of $U$ in $M$ and the already mentioned fact that $\bigcup_{n \geq 1} \Gamma_{n}$ is dense in $C\left(2^{\mathbb{N}} ; M\right)$ that $\bigcup_{n \geq 1} \Gamma_{n}(U)$ is dense in $C\left(2^{\mathbb{N}} ; M\right)$ (and a subset of $\left\{\gamma \in C\left(2^{\mathbb{N}} ; M\right)\right.$ : $\left.\left.\gamma\left(2^{\mathbb{N}}\right) \subseteq U\right\}\right)$.

(ii) Let $V:=U \cup M \backslash \bar{U}$ (which is a dense open subset of $M$ ), and for each $n \in \mathbb{N}$, let

$$
\Gamma_{n}^{*}:=\left\{\gamma \in \Gamma_{n}(V):(g \circ \gamma)(t) \neq(g \circ \gamma)\left(t^{\prime}\right) \text { if } t, t^{\prime} \in \gamma^{-1}(U) \text { and }\left.t\right|_{n} \neq\left. t^{\prime}\right|_{n}\right\}
$$

It is easy to check that for each $n \in \mathbb{N}, \bigcup_{k \geq n} \Gamma_{k}^{*}$ is dense in $C\left(2^{\mathbb{N}} ; M\right)$. Now, for each $n \in \mathbb{N}$ and $\hat{\gamma} \in \Gamma_{n}^{*}$, choose $r_{n}(\hat{\gamma})>0$ so that

(i) $B\left(\hat{\gamma}(t) ; r_{n}(\hat{\gamma})\right) \subseteq U$ for all $t \in \hat{\gamma}^{-1}(U)$;

(ii) $B\left(\hat{\gamma}(t) ; r_{n}(\hat{\gamma})\right) \subseteq M \backslash \bar{U}$ for all $t \in \hat{\gamma}^{-1}(M \backslash \bar{U})$;

(iii) $g\left(B\left(\hat{\gamma}(t) ; r_{n}(\hat{\gamma})\right)\right) \cap g\left(B\left(\hat{\gamma}\left(t^{\prime}\right) ; r_{n}(\hat{\gamma})\right)\right)=\varnothing$ for all $t, t^{\prime} \in \hat{\gamma}^{-1}(U)$ such that $\left.t\right|_{n} \neq$ $\left.t^{\prime}\right|_{n}$.

One can now check that the set

$$
\bigcap_{n \in \mathbb{N}}\left(\bigcup_{k \geq n}\left\{\gamma \in C\left(2^{\mathbb{N}} ; M\right) \text { : there exists a } \hat{\gamma} \in \Gamma_{k}^{*} \text { with } \max _{t \in 2^{\mathbb{N}}}[d(\gamma(t), \hat{\gamma}(t))]<r_{k}(\hat{\gamma})\right\}\right)
$$

is residual in $C\left(2^{\mathbb{N}} ; M\right)$ and has the desired properties.

From the previous lemma, we can deduce that for any proper $\sigma$-ideal $\mathscr{A}$ of subsets of $2^{\mathbb{N}}$, the $\mathscr{A}$-negligible sets form a $\sigma$-ideal of subsets of $M$ that contains all the first category subsets of $M$. In addition, if $M$ is a complete metric space and $N \subseteq M$ has the Baire property, then one can show that $N$ is $\mathscr{A}$-negligible if and only if $N$ is first category in $M$. Thus, the interesting $\mathscr{A}$-negligible sets are necessarily among those subsets of $M$ that are not very topologically respectable.

Given a proper $\sigma$-ideal $\mathscr{A}$ of subsets of $2^{\mathbb{N}}$ and a topological space $X$, we say that $X$ is nearly Stegall with respect to $\mathscr{A}$ if for every complete metric space $M$ and minimal usco $\varphi: M \rightarrow 2^{X},\{x \in M: \varphi(x)$ is not a singleton $\}$ is $\mathscr{A}$-negligible. Thus, for any proper $\sigma$ ideal $\mathscr{A}$ of subsets of $2^{\mathbb{N}}$ and any topological space $X$, if $X$ is nearly Stegall with respect to $\mathcal{A}$, then $X$ is weakly Stegall.

As with Proposition 3.1, the proof of the following result is identical to that given in [2, Theorem 3.1.5] and thus not presented here.

Proposition 3.7. Let $X$ and $Y$ be topological spaces and $\mathscr{A}$ a proper $\sigma$-ideal of subsets of $2^{\mathbb{N}}$. 
(i) Let $g: X \rightarrow Y$ be a perfect mapping onto $Y$. If $X$ is nearly Stegall with respect to $\mathscr{A}$, then $Y$ is nearly Stegall with respect to $A$.

(ii) Let $\left\{X_{n}: n \in \mathbb{N}\right\}$ be a cover of $X$. If each $X_{n}$ is closed and nearly Stegall with respect to $\mathscr{A}$, then $X$ is nearly Stegall with respect to $\mathscr{A}$.

(iii) If $\left\{X_{n}: n \in \mathbb{N}\right\}$ are nearly Stegall with respect to $\mathscr{A}$, then $\pi_{n=1}^{\infty} X_{n}$ is nearly Stegall with respect to $\mathscr{A}$.

By combining Riesz's representation theorem with Proposition 3.7, we obtain the following fact.

Corollary 3.8. Let $\mathscr{A}$ be a proper $\sigma$-ideal of subsets of $2^{\mathbb{N}}$. Then for a compact Hausdorff space $K,\left(C(K)^{*}\right.$, weak $\left.{ }^{*}\right)$ is nearly Stegall with respect to $\mathscr{A}$ if and only if $\mathcal{M}_{1}^{+}(K)$ is nearly Stegall with respect to $\mathscr{A}$.

Theorem 3.9. Let $\mathscr{A}$ be a proper $\sigma$-ideal of subsets of $2^{\mathbb{N}}$ and let $A$ be any subset of $(0,1)$ such that $\gamma^{-1}(A) \in \mathscr{A}$ for each homeomorphic embedding of $2^{\mathbb{N}}$ into $[0,1]$. Then $\left(C\left(K_{A}\right)^{*}\right.$, weak $\left.^{*}\right)$ is nearly Stegall with respect to $\mathscr{A}$. In particular, $\left(C\left(K_{A}\right),\|\cdot\|_{\infty}\right)$ is a Gâteaux differentiability space.

Proof. By Corollary 3.8, it is sufficient to show that $M_{1}^{+}\left(K_{A}\right)$ is nearly Stegall with respect to $\mathscr{A}$. To this end, let $\varphi: M \rightarrow 2^{\mathcal{M}_{1}^{+}\left(K_{A}\right)}$ be a minimal usco acting from a complete metric space $M$ into $M_{1}^{+}\left(K_{A}\right)$. Furthermore, let $\left(g_{n}: n \in \mathbb{N}\right)$ and $G$ be the continuous functions and dense $G_{\delta}$ subset of $M$ given in Lemma 2.4. Since $C\left(2^{\mathbb{N}} ; G\right)$ is a residual subspace of $C\left(2^{\mathbb{N}} ; M\right)$ and the restriction of $\varphi$ to $G$ is still a minimal usco (see, Proposition 1.2(ii)), there is no loss of generality in assuming that $M=G$. Now, by Theorem 2.5, it is sufficient to show that for each $n \in \mathbb{N}$, the complement of the set

$$
G_{n}:=\left\{x \in M: \text { the restriction of all the measures in } \varphi(x) \text { to } \pi_{A}^{-1}\left(g_{n}(x)\right) \text { coincide }\right\}
$$

is $\mathscr{A}$-negligible in $M$. To this end, we fix $n \in \mathbb{N}$ and let the $\mathscr{G}_{n}$ be the union of all the open subsets of $M$ on which $g_{n}$ is constant and let $\mathscr{H}_{n}:=M \backslash \overline{\mathscr{G}_{n}}$. By Lemma 2.7, we know that $\mathscr{G}_{n} \backslash G_{n}$ is first category in $M$ and so $\mathscr{A}$-negligible. Hence we need only show that $\mathscr{H}_{n} \backslash G_{n}$ is $\mathscr{A}$-negligible since $M \backslash\left(\mathscr{G}_{n} \cup \mathscr{H}_{n}\right)$ is a closed nowhere dense subset of $M$ and thus an $\mathscr{A}$-negligible subset. In fact, since $\mathscr{H}_{n} \backslash G_{n} \subseteq g_{n}^{-1}(A) \cap \mathscr{H}_{n}$, we need only to show that $g_{n}^{-1}(A) \cap \mathscr{H}_{n}$ is $\mathscr{A}$-negligible. By Lemma 3.6, the set of all $\gamma \in C\left(2^{\mathbb{N}} ; M\right)$ for which (i) $\gamma\left(2^{\mathbb{N}}\right) \subseteq \mathscr{G}_{n} \cup \mathcal{H}_{n}$ and (ii) $\left(g_{n} \circ \gamma\right)$ is 1 -to- 1 on $D_{\gamma}^{n}:=\gamma^{-1}\left(\mathscr{H}_{n}\right)$ is residual in $C\left(2^{\mathbb{N}} ; M\right)$. For any such $\gamma$ we have

$$
\gamma^{-1}\left(g_{n}^{-1}(A) \cap \mathscr{H}_{n}\right)=\left(g_{n} \circ \gamma\right)^{-1}(A) \cap D_{\gamma}^{n} \in \mathscr{A}
$$

since (i) $\left(g_{n} \circ \gamma\right)$ is 1-to- 1 on the clopen set $D_{\gamma}^{n}$ and (ii) every continuous 1-to-1 mapping from a clopen subset of $2^{\mathbb{N}}$ into $[0,1]$ can be extended to be a homeomorphic embedding of $2^{\mathbb{N}}$ into $[0,1]$. Thus, $g_{n}^{-1}(A) \cap \mathscr{H}_{n}$ is $\mathscr{A}_{\text {-negligible. }}$

Lemma 3.10. There exists an everywhere second category subset $A$ of $(0,1)$ and a proper $\sigma$ ideal $\mathscr{A}$ of subsets of $2^{\mathbb{N}}$ such that $\gamma^{-1}(A) \in \mathscr{A}$ for each homeomorphic embedding $\gamma: 2^{\mathbb{N}} \rightarrow$ $[0,1]$. 
Proof. Let $\kappa$ be the least ordinal of cardinality $2^{\mathrm{N}_{0}}$, let $\left\{\left(\gamma_{n}^{\alpha}: n \in \mathbb{N}\right): \alpha<\kappa\right\}$ be an enumeration of all the sequences of continuous one-to-one functions from $2^{\mathbb{N}}$ into $[0,1]$ and let $\left\{E^{\alpha}: \alpha<\kappa\right\}$ be an enumeration of all the nonmeagre Borel subsets of $(0,1)$. Inductively, we may choose $a_{\alpha} \in E^{\alpha} \backslash\left\{\gamma_{n}^{\beta}\left(x_{\beta}\right): n \in \mathbb{N}\right.$ and $\left.\beta<\alpha\right\}$ and $x_{\alpha} \in 2^{\mathbb{N}}$ such that $\gamma_{n}^{\alpha}\left(x_{\alpha}\right) \neq a_{\beta}$ for any $n \in \mathbb{N}$ and $\beta \leq \alpha$.

Set $A:=\left\{a_{\alpha}: \alpha<\kappa\right\}$. Then $A$ is everywhere second category in $(0,1)$ and for any sequence $\left(\gamma_{n}: n \in \mathbb{N}\right)$ of continuous one-to-one functions from $2^{\mathbb{N}}$ into $[0,1],\left(\gamma_{n}^{-1}(A)\right.$ : $n \in \mathbb{N}$ ) does not form a cover of $2^{\mathbb{N}}$. So, if we take $\mathscr{A}$ to be the $\sigma$-ideal generated by the inverse images, $\gamma^{-1}(A)$, as $\gamma$ runs over all the continuous one-to-one functions from $2^{\mathbb{N}}$ into $[0,1]$, then $\mathscr{A}$ will be a proper $\sigma$-ideal of subsets of $2^{\mathbb{N}}$ such that $\gamma^{-1}(A) \in \mathscr{A}$ for each 1-to-1 mapping $\gamma$ from $2^{\mathbb{N}}$ into $[0,1]$.

Corollary 3.11. There exists a Banach space $(X,\|\cdot\|)$ such that $\left(X^{*}\right.$, weak $\left.{ }^{*}\right)$ is weakly Stegall but $(X,\|\cdot\|)$ is not weak Asplund. In particular, $(X,\|\cdot\|)$ is a Gâteaux differentiability space that is not weak Asplund.

Proof. Let $A$ be the set constructed in Lemma 3.10 and let $\mathscr{A}$ be the corresponding $\sigma$ ideal on $2^{\mathbb{N}}$. Then $A$ satisfies the hypotheses of Theorem 3.9 with respect to $\mathscr{A}$. Hence $\left(C\left(K_{A}\right)\right.$, weak $\left.{ }^{*}\right)$ is nearly Stegall with respect to $\mathscr{A}$ and so weakly Stegall. On the other hand, if $\left(C\left(K_{A}\right),\|\cdot\|_{\infty}\right)$ is weak Asplund, then by [1], every closed subset of $K_{A}$ contains a dense completely metrizable subspace. However, by Theorem 2.2 this implies that $A$ is meagre (in fact perfectly meagre), which is not the case. Therefore, $\left(C\left(K_{A}\right),\|\cdot\|_{\infty}\right)$ is not weak Asplund.

3.1. Open problems. (i) Is $\left(C\left(K_{A}\right),\|\cdot\|_{\infty}\right)$ weak Asplund if and only if $A$ is perfectly meagre? Certainly, if $\left(C\left(K_{A}\right),\|\cdot\|_{\infty}\right)$ is weak Asplund, then $A$ must be perfectly meagre. On the other hand, it follows from Theorem 3.9 that if $A$ is perfectly meagre, then $\left(C\left(K_{A}\right),\|\cdot\|_{\infty}\right)$ is almost weak Asplund.

(ii) Is every Gâteaux differentiability space almost weak Asplund? Our example from Corollary 3.11 does not answer this question as it is almost weak Asplund. The natural candidate for a counterexample to this question is the space $\left(C\left(K_{(0,1)}\right),\|\cdot\|_{\infty}\right)$ which is not almost weak Asplund (as the supremum norm is Gâteaux differentiable only on a first category subset of $\left.C\left(K_{(0,1)}\right)\right)$ but which may well be a Gâteaux differentiability space.

\section{Acknowledgment}

The author would like to thank both David Fremlin and David Preiss for (independently) bringing Lemma 3.10 to his attention. The author would also like to thank David Preiss for significantly improving and simplifying the presentation of the paper.

\section{References}

[1] M. M. Čoban and P. S. Kenderov, Generic Gâteaux differentiability of convex functionals in $C(T)$ and the topological properties of $T$, Proceedings of 15th Spring Conference of the Union of Bulgarian Mathematicians, SljanĈev Brjag, 1986, pp. 141-149.

[2] M. J. Fabian, Gâteaux Differentiability of Convex Functions and Topology: Weak Asplund Spaces, Canadian Mathematical Society Series of Monographs and Advanced Texts, John Wiley \& Sons, New York, 1997. 
[3] O. F. K. Kalenda, Stegall compact spaces which are not fragmentable, Topology Appl. 96 (1999), no. 2, 121-132.

[4] - A weak Asplund space whose dual is not in Stegall's class, Proc. Amer. Math. Soc. 130 (2002), no. 7, 2139-2143.

[5] P. S. Kenderov, W. B. Moors, and S. Sciffer, A weak Asplund space whose dual is not weak fragmentable, Proc. Amer. Math. Soc. 129 (2001), no. 12, 3741-3747.

[6] W. B. Moors and S. Somasundaram, Some recent results concerning weak Asplund spaces, Acta Univ. Carolin. Math. Phys. 43 (2002), no. 2, 67-86.

Warren B. Moors: Department of Mathematics, The University of Auckland, Private Bag 92019, Auckland, New Zealand

E-mail address: moors@math.auckland.ac.nz 


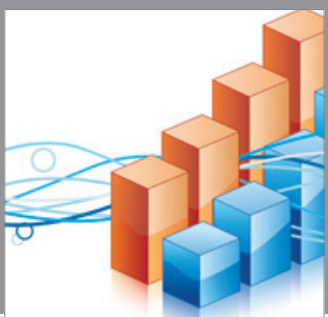

Advances in

Operations Research

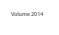

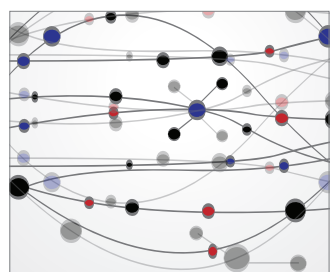

\section{The Scientific} World Journal
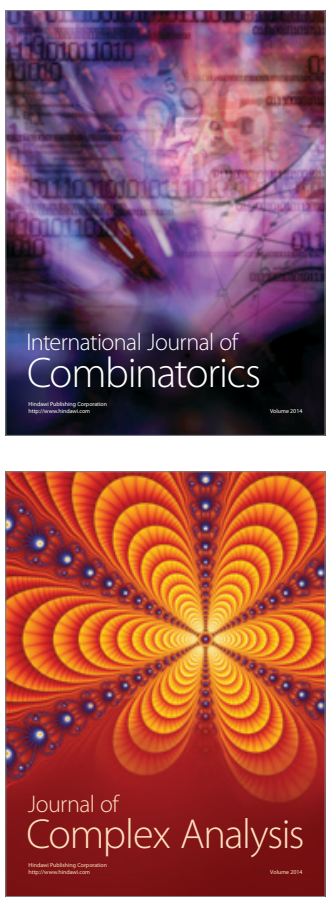

International Journal of

Mathematics and

Mathematical

Sciences
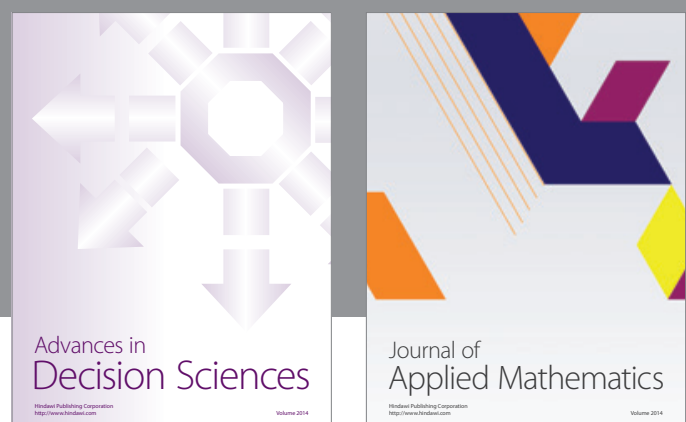

Journal of

Applied Mathematics
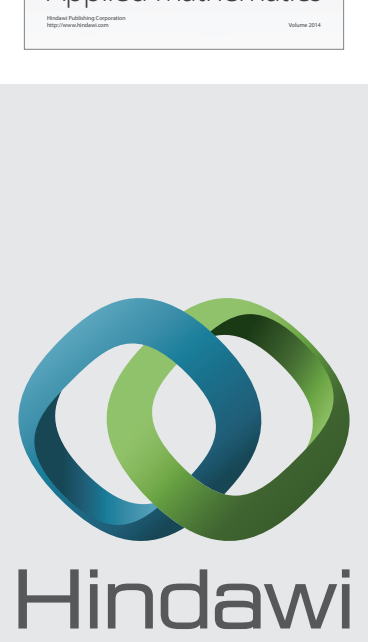

Submit your manuscripts at http://www.hindawi.com
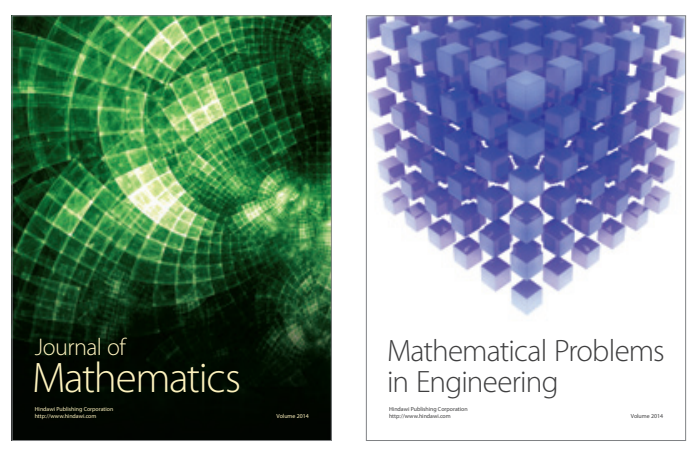

Mathematical Problems in Engineering
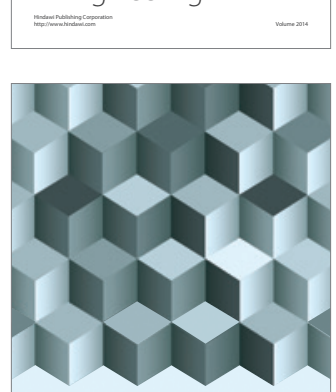

Journal of

Function Spaces
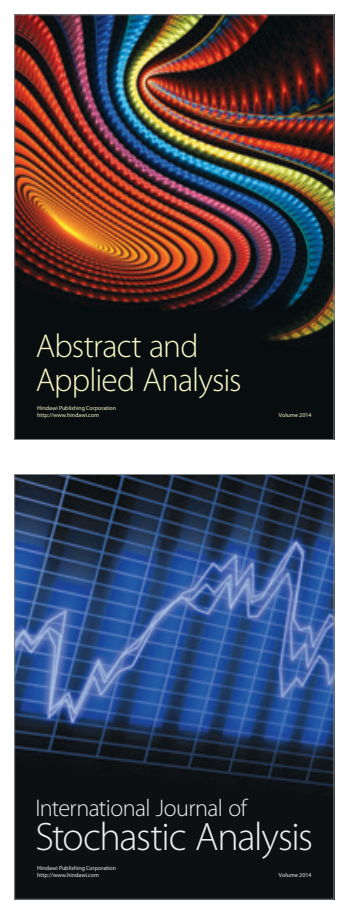

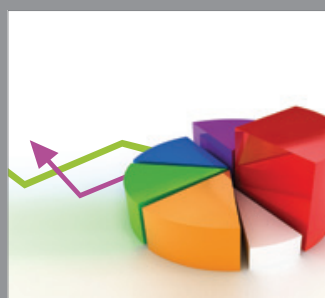

ournal of

Probability and Statistics

Promensencen
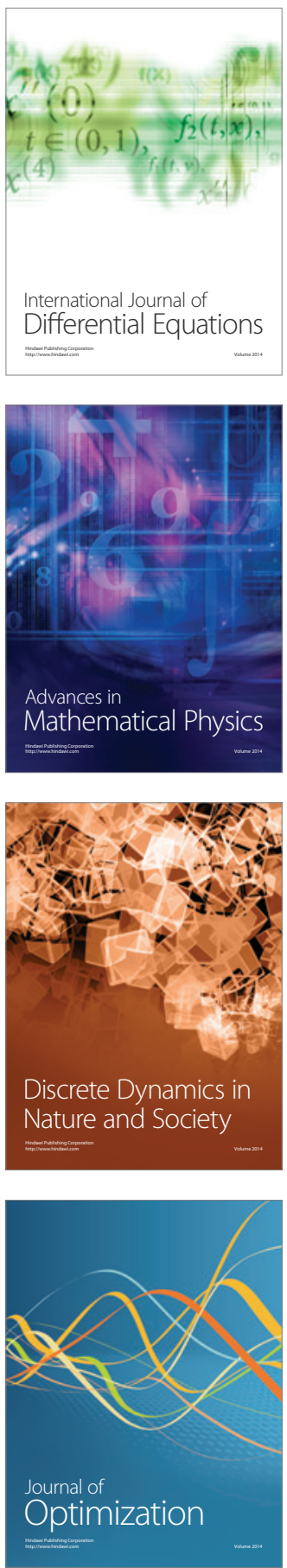